\title{
Design and implementation of an electro-opijc: l] backplane with pluggable in-plane connectors
}

Speaker:

Richard Pitwon

Xyratex Technology Ltd

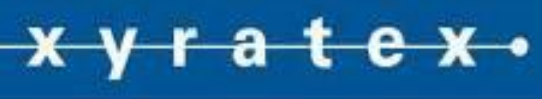

SPIE Photonics West - OPTO

Paper: Opto 7607-18

San Francisco

Tuesday $26^{\text {th }}$ January 2010
R. Pitwon, K. Hopkins

K. Wang, D. R. Selviah, H. Baghsiahi,

B. Offrein, R. Dangel, F. Horst,

M. Halter, Max Gmür
Xyratex Technology Ltd

University College London IBM Zürich

Vario-optics AG 


\section{Overview}

․ Design challenges in data storage systems

Active in-plane optical backplane connector

ㅁ Demonstration platform
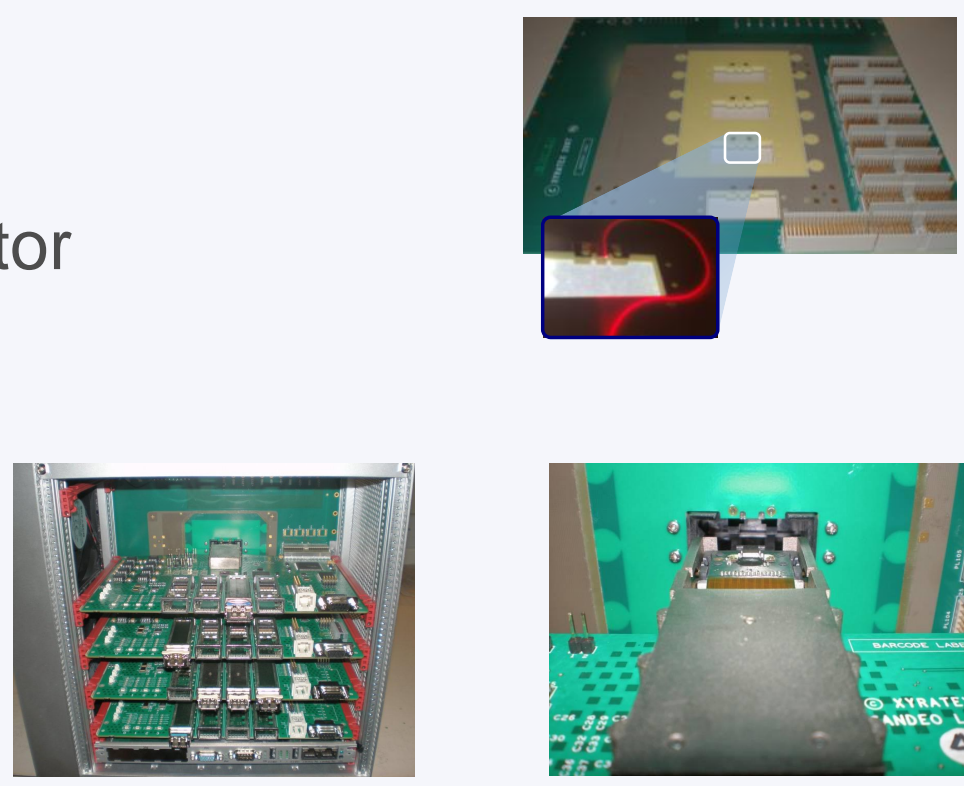

口 Electro-optical backplane with polymer waveguides

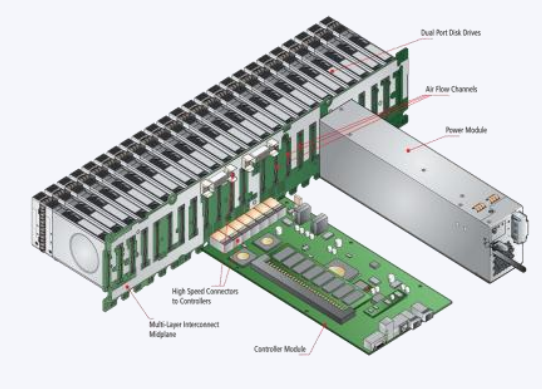




\section{Design challenges in modern data storage systems}

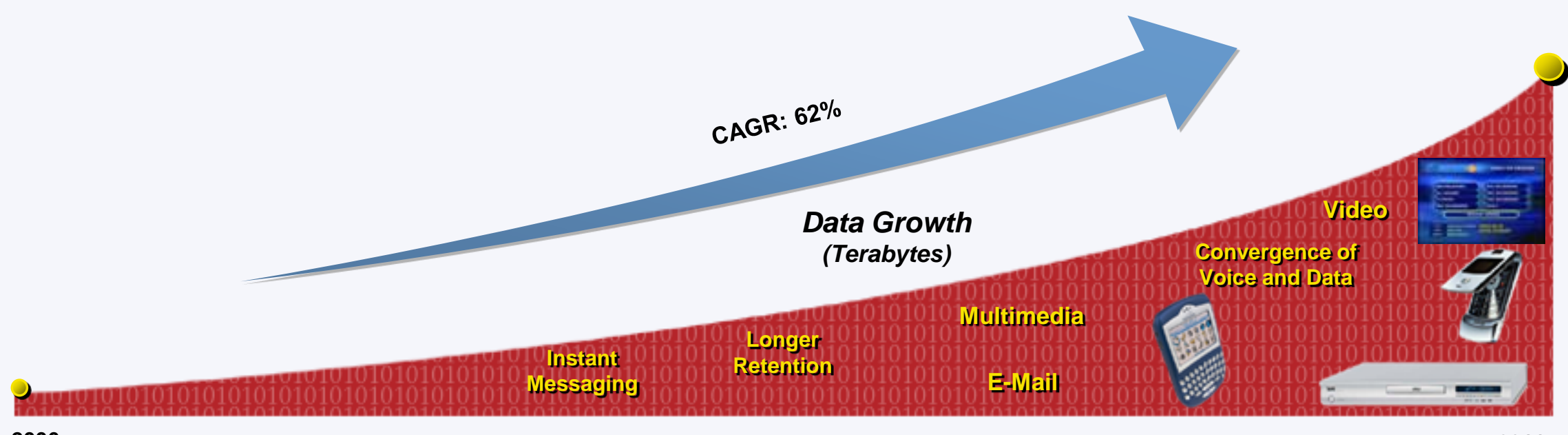




\section{Data storage protocol and form factor trends}

Disk drive form factors decreasing

3.5" HDD

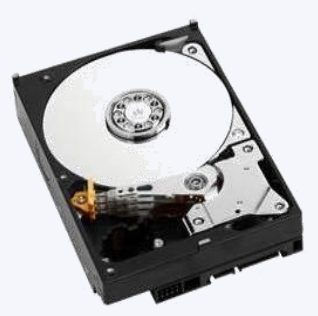

2.5" HDD

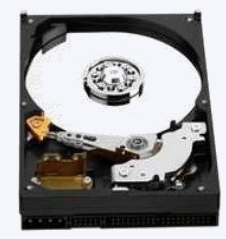

$2.5 "$ SSD

$1.8 "$ SSD
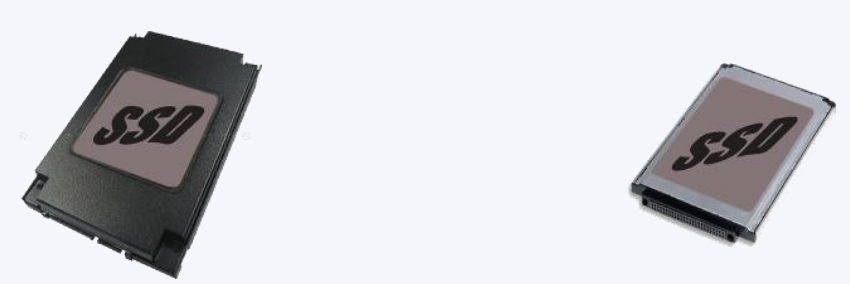

Data storage interconnect speeds increasing

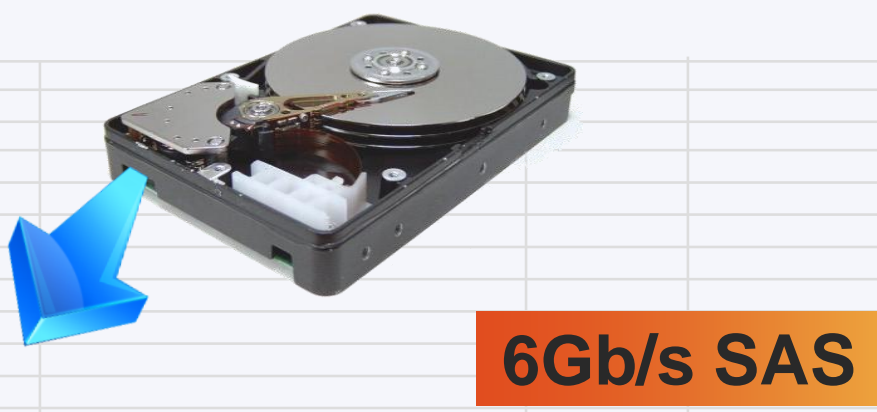

$3 \mathrm{~Gb} / \mathrm{s}$ SAS

$\begin{array}{lllllllllllll}2003 & 2004 & 2005 & 2006 & 2007 & 2008 & 2009 & 2010 & 2011 & 2012 & 2013 & 2014 & 2015\end{array}$

Source: SCSI Trade Association Sep $08 \quad$ www.scsita.org 


\section{Data storage array backplane topology}

$\underline{\text { Redundant dual star architecture }}$

Data storage devices

a Controller modules

G Midplane interconnect

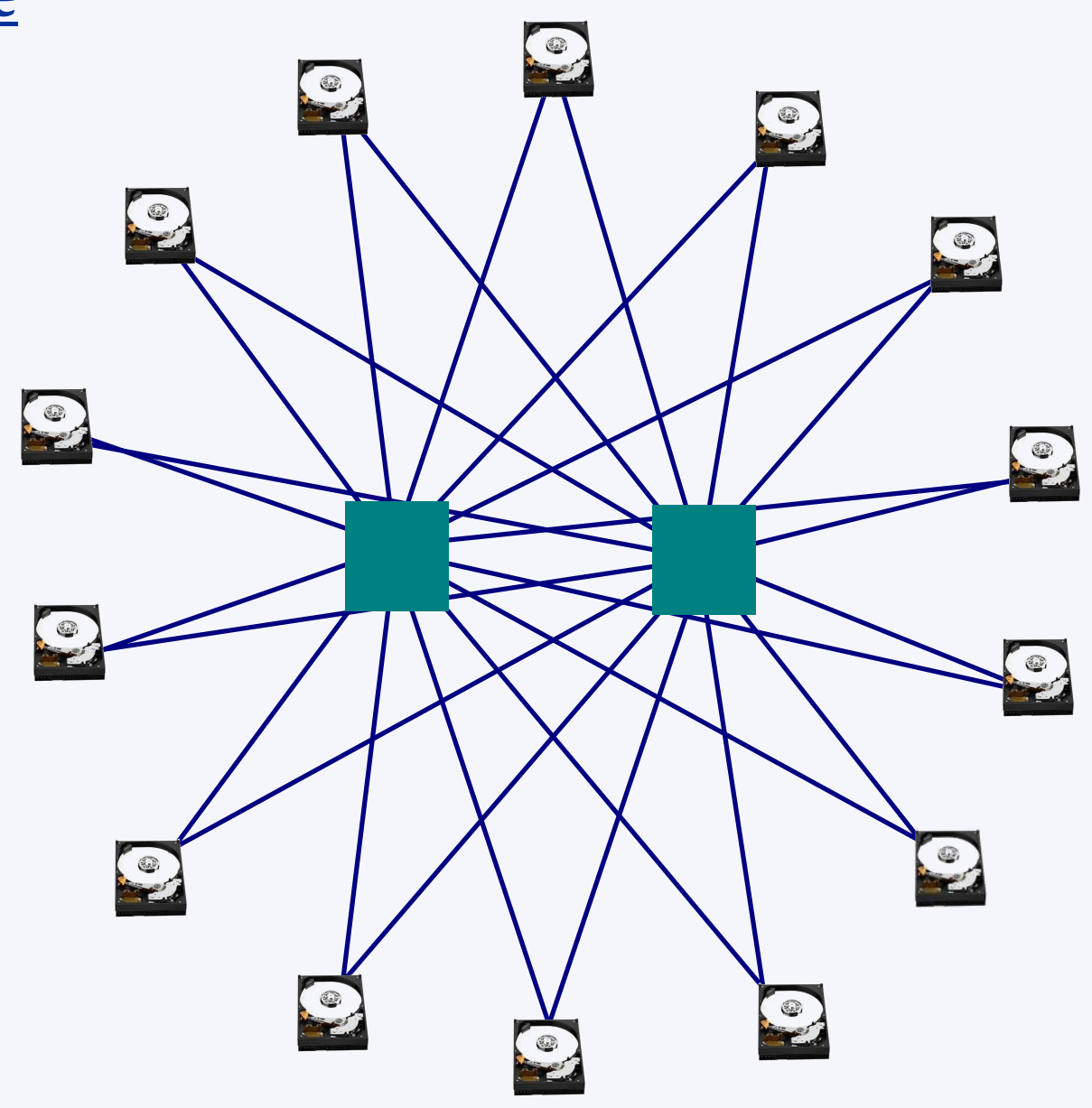




\section{Design and performance constraints}

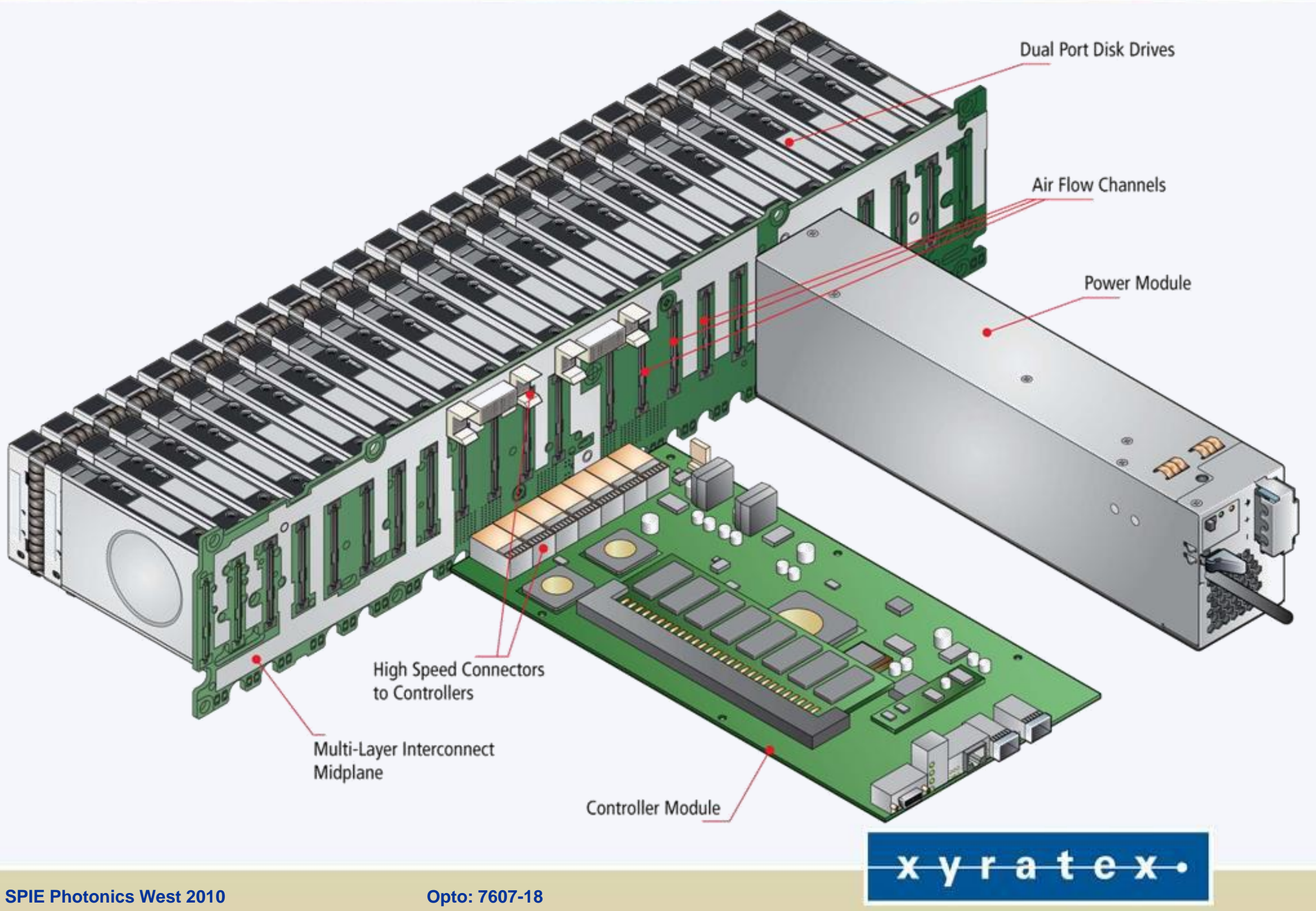




\section{Design and performance constraints}

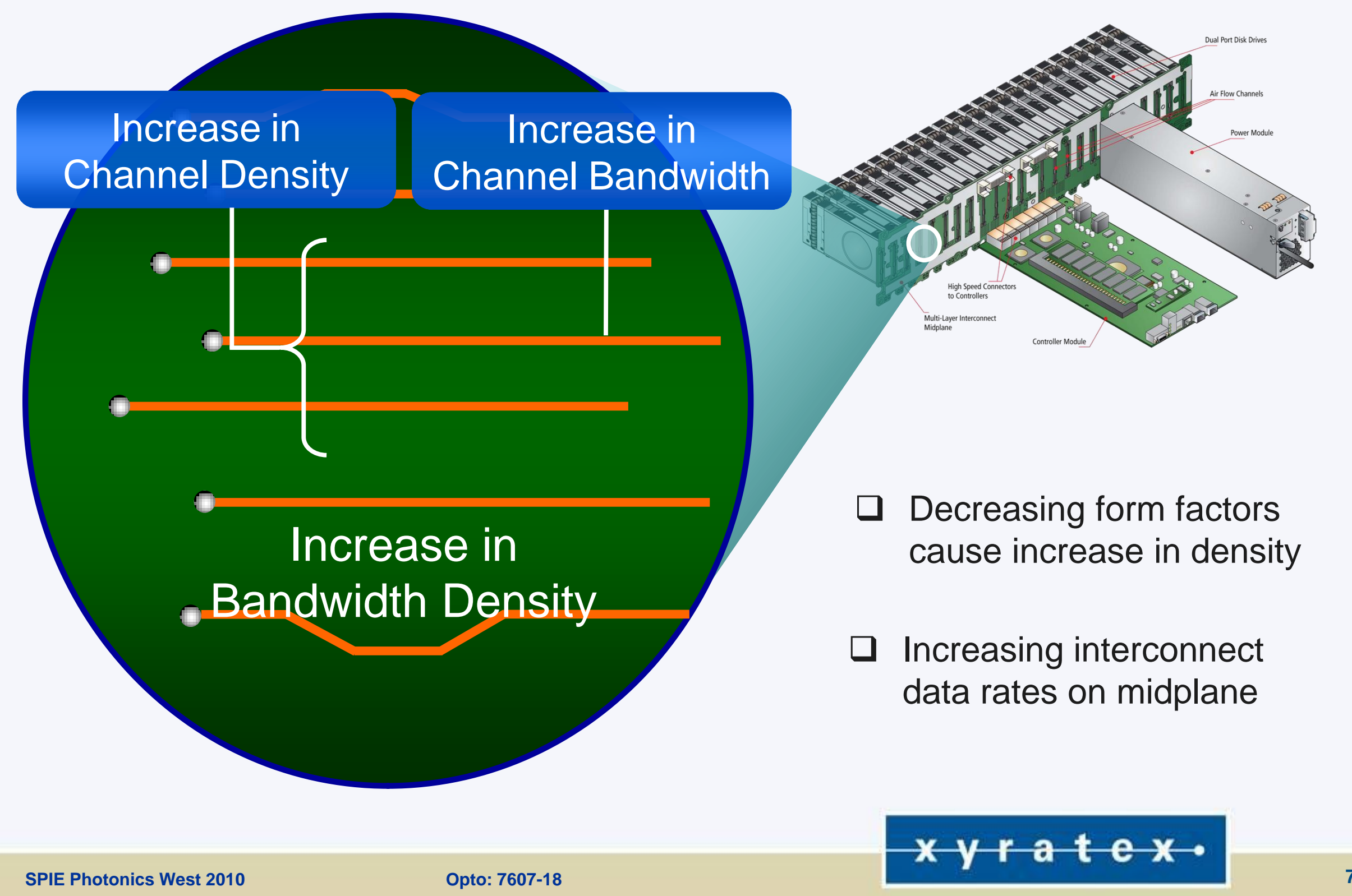




\section{Design and performance constraints}

Copper layers for power distribution

Copper layers for low speed communication

$\square$ Optical layers for high speed communication

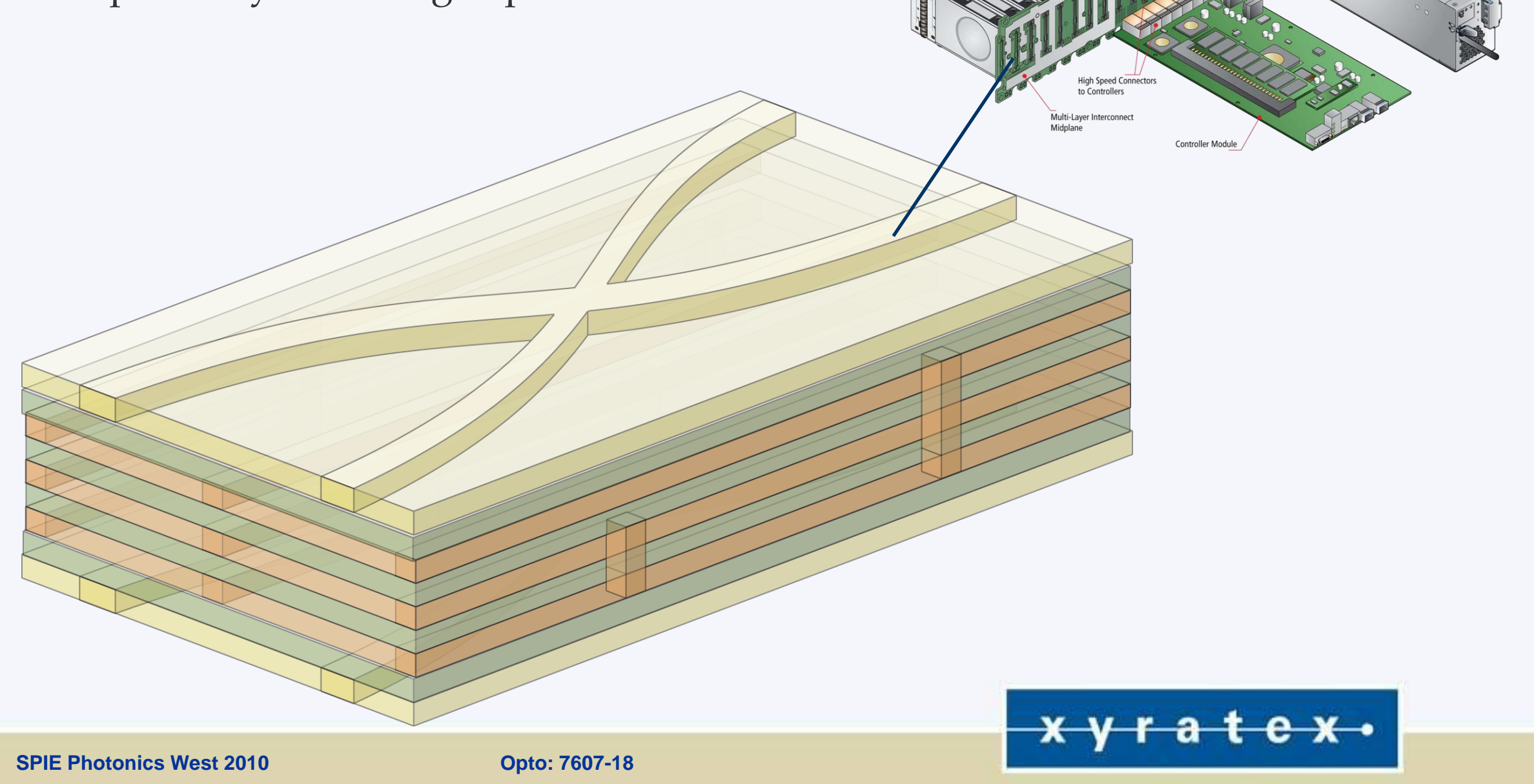




\section{Electro-optical backplane with polymer waveguides}
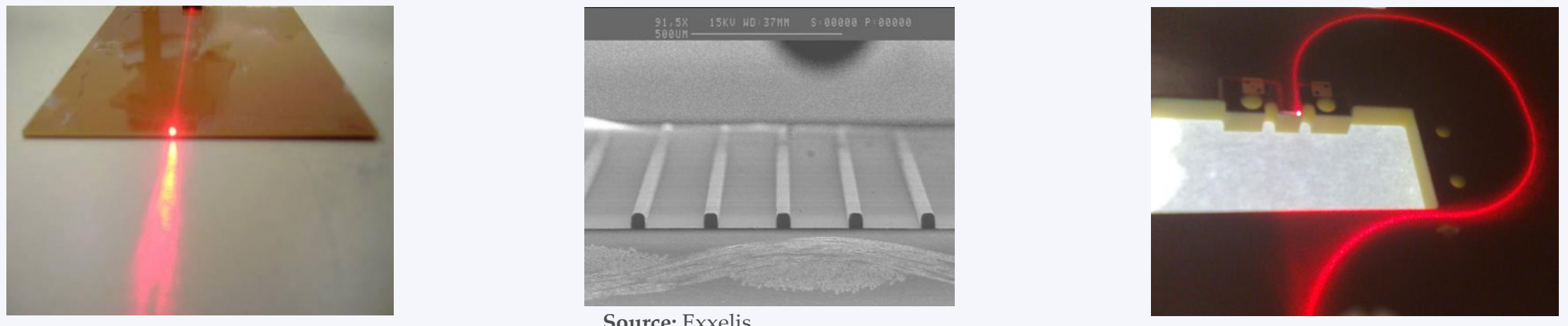


\section{Polymer optical waveguide layer}

Optical polymer

- Low loss at $850 \mathrm{~nm}$

\section{Waveguide} characteristics

$\square \mathrm{n}_{\text {core }}$

$\square \mathrm{n}_{\text {cladding }}$

$\square \Delta \mathrm{n}$

$\square$ N.A.

Core dimensions

$$
\begin{aligned}
& =1.56 \\
& =1.524 \\
& =2.3 \% \\
& =0.33
\end{aligned}
$$
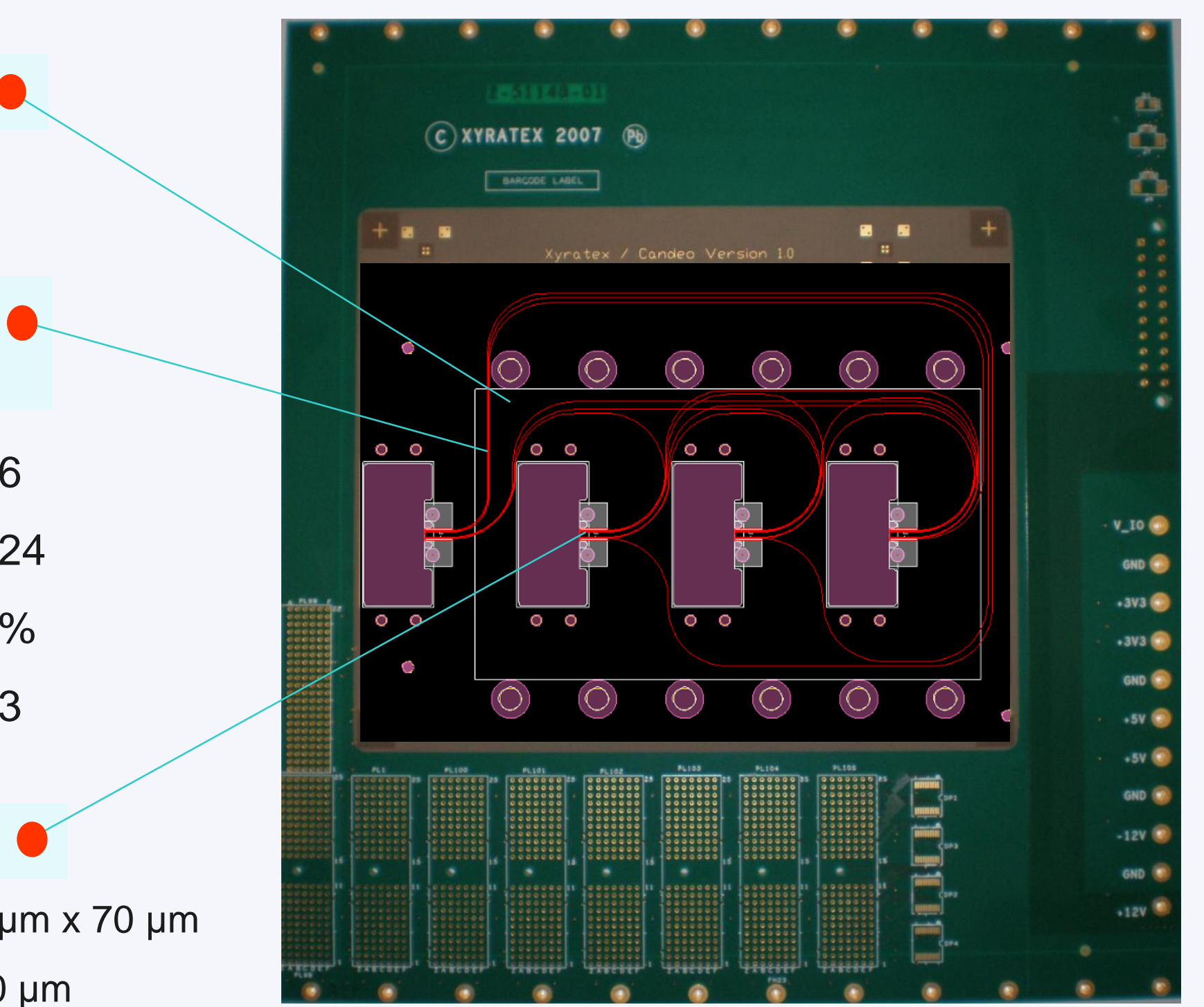

口 Cross-section $=70 \mu \mathrm{m} \times 70 \mu \mathrm{m}$

口 Pitch (centre) $=250 \mu \mathrm{m}$ 


\section{Optical interconnect design}

Waveguide layout

- UCL

] Xyratex

口 IBM Zurich
Crossovers

Non-orthogonal

$130^{\circ}$ to $160^{\circ}$
Min. Pitch

口 $250 \mu \mathrm{m}$

\section{Bends}

a $\mathrm{RoC}=17 \mathrm{~mm}$

$\square$ Negative and positive cascades

\section{Engagement apertures}

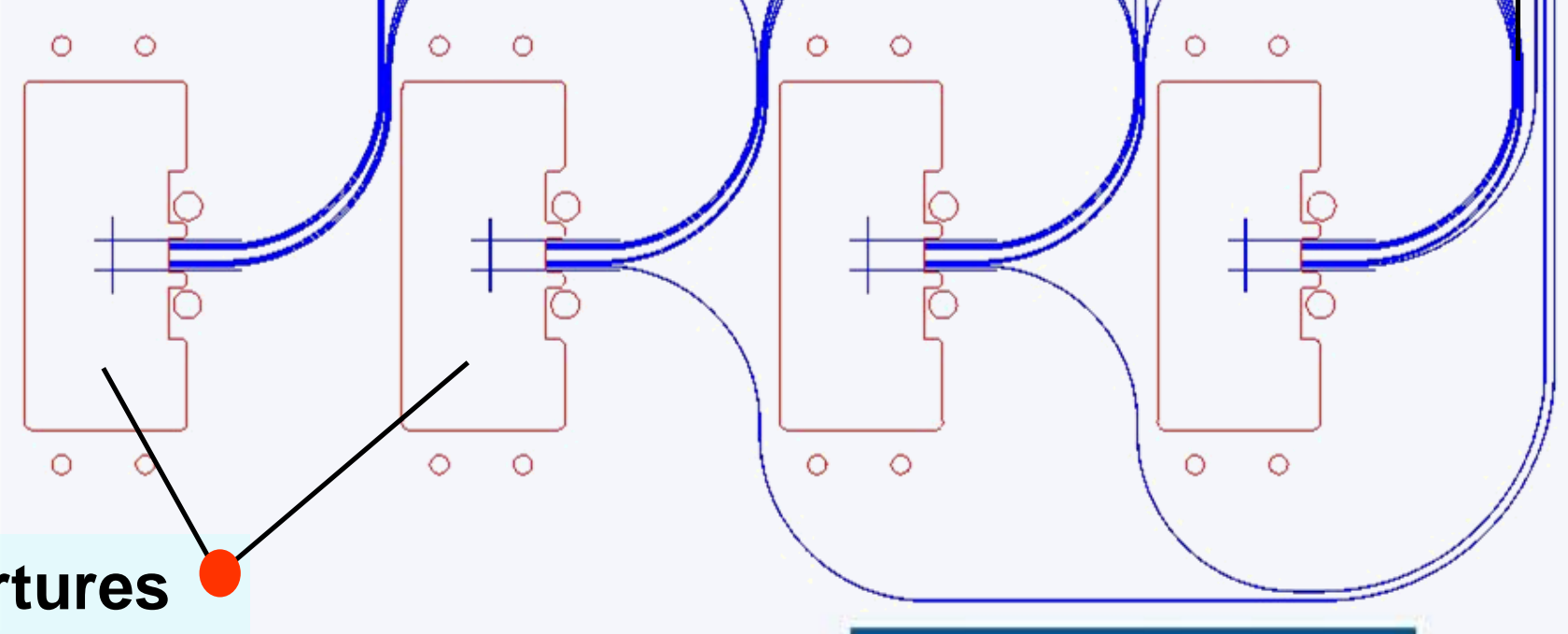




\section{Electro-optical PCB fabrication process}

1. Deposit lower refractive index polymer (cladding) onto substrate surface

2. Cure polymer layer with exposure to ultra-violet light to harden

3. Deposit higher refractive index (core) polymer onto lower cladding layer

4. Align UV laser write head into position

5. Activate laser and move write head to pattern waveguide features in core layer

6. Remove uncured portions of the con layer

7. Deposit lower refractive index polyr er onto patterned core layer

8. Cure upper cladding layer with UV Ifght

\section{UV Exposure}

Upper cladding

Core layer

Lower cladding

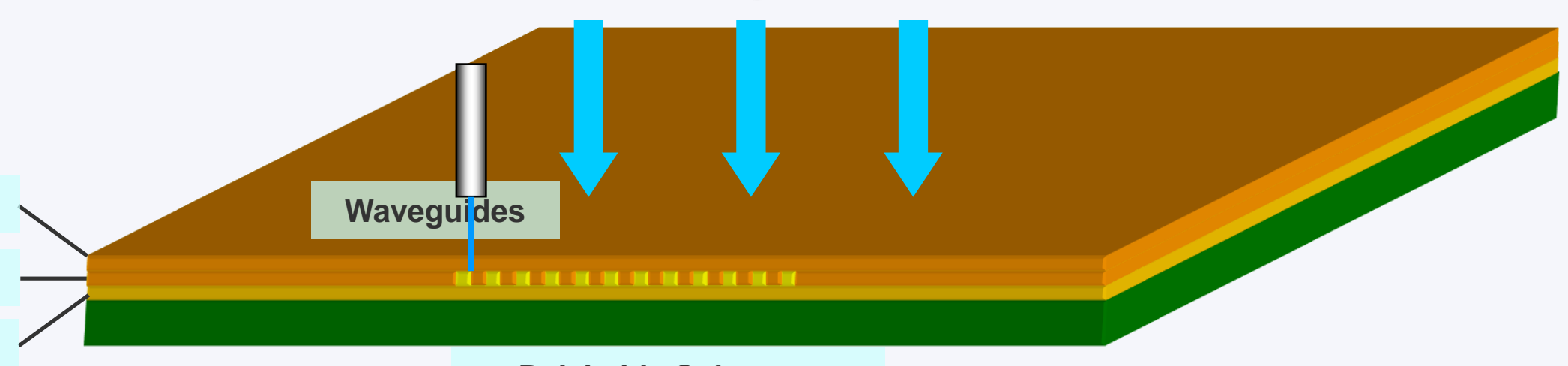

Polyimide Substrate 


\section{Electro-optical midplane}

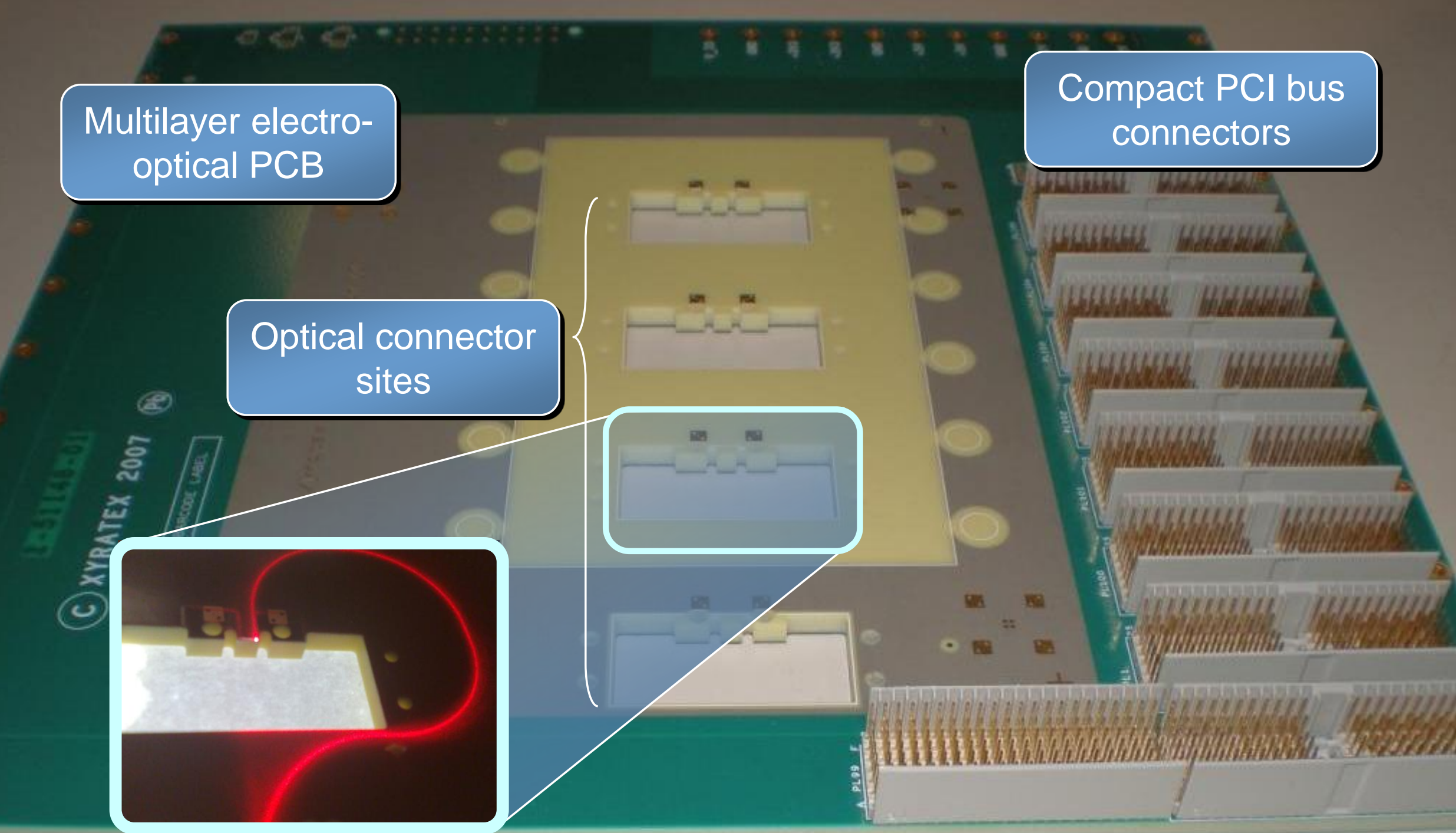




\section{Passive alignment and assembly}

\section{Mechanical registration features}

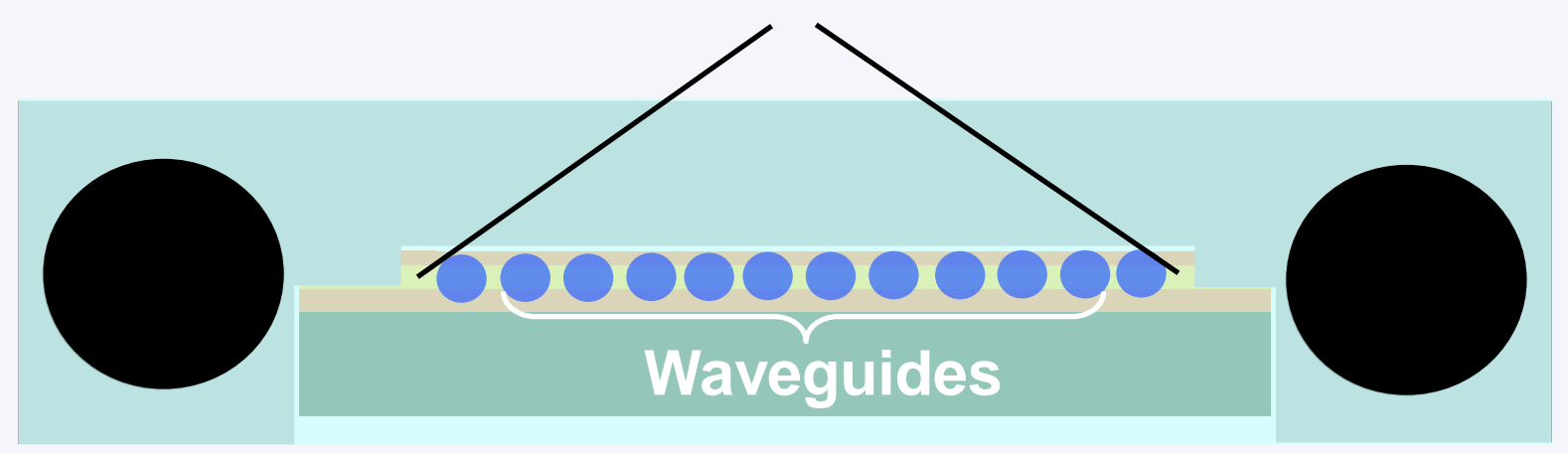

$\square$ Deposit lower cladding layer

$\square$ Deposit core layer

- Pattern core layer including registration waveguides

$\square$ Deposit upper cladding layer

Remove part of upper cladding for mechanical access

Align MT compliant receptacle with microlens array
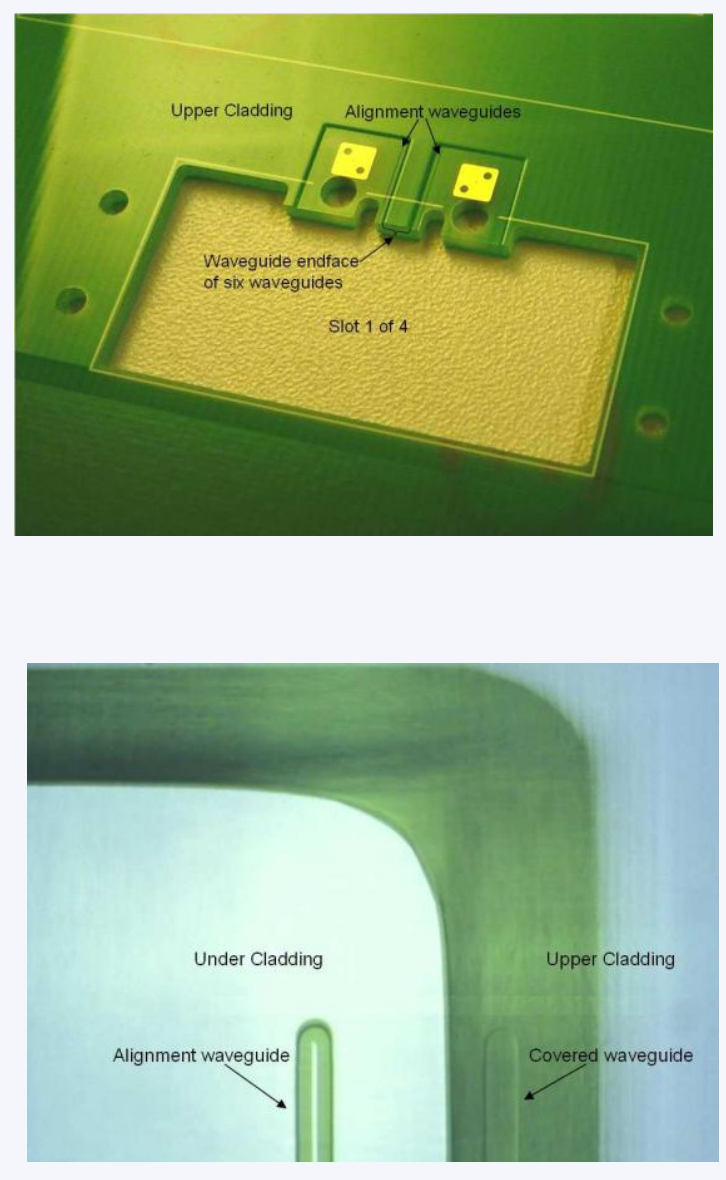


\section{Optical connector receptacles}

Primary receptacle

- Low tolerance

- Pluggable connector

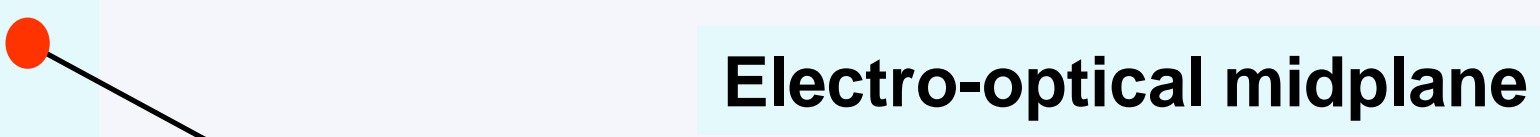

Secondary receptacle

- High tolerance

- Lens holder

口 MT compliant

Electro-optical midplane
Microlens array

․ MT compliant
Connector site 
Waveguide insertion loss measurements

$\square$ Measured loss without index matching fluid

- Measured loss with index matching fluid

- Calculated loss
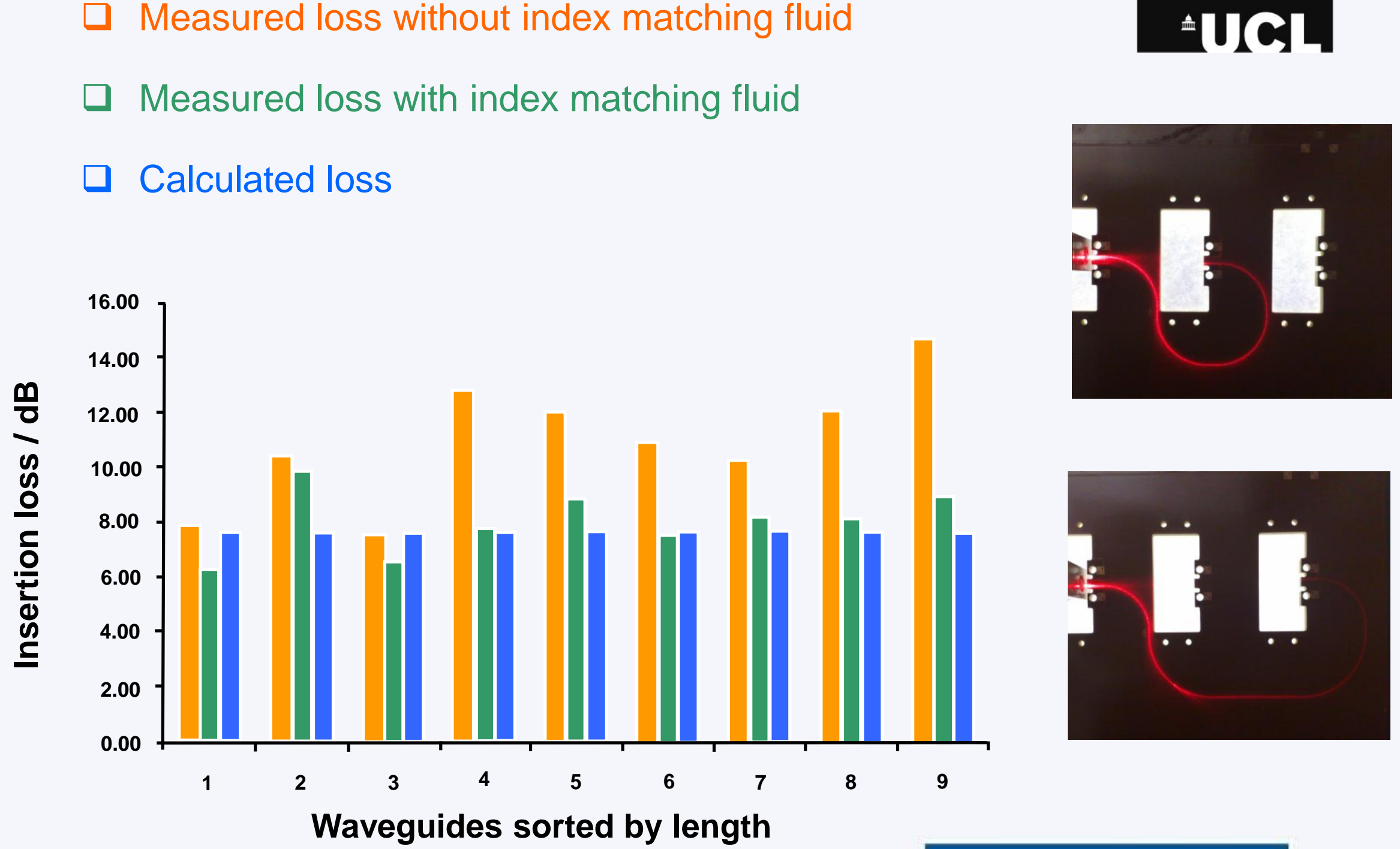


\section{Active optical backplane connector}
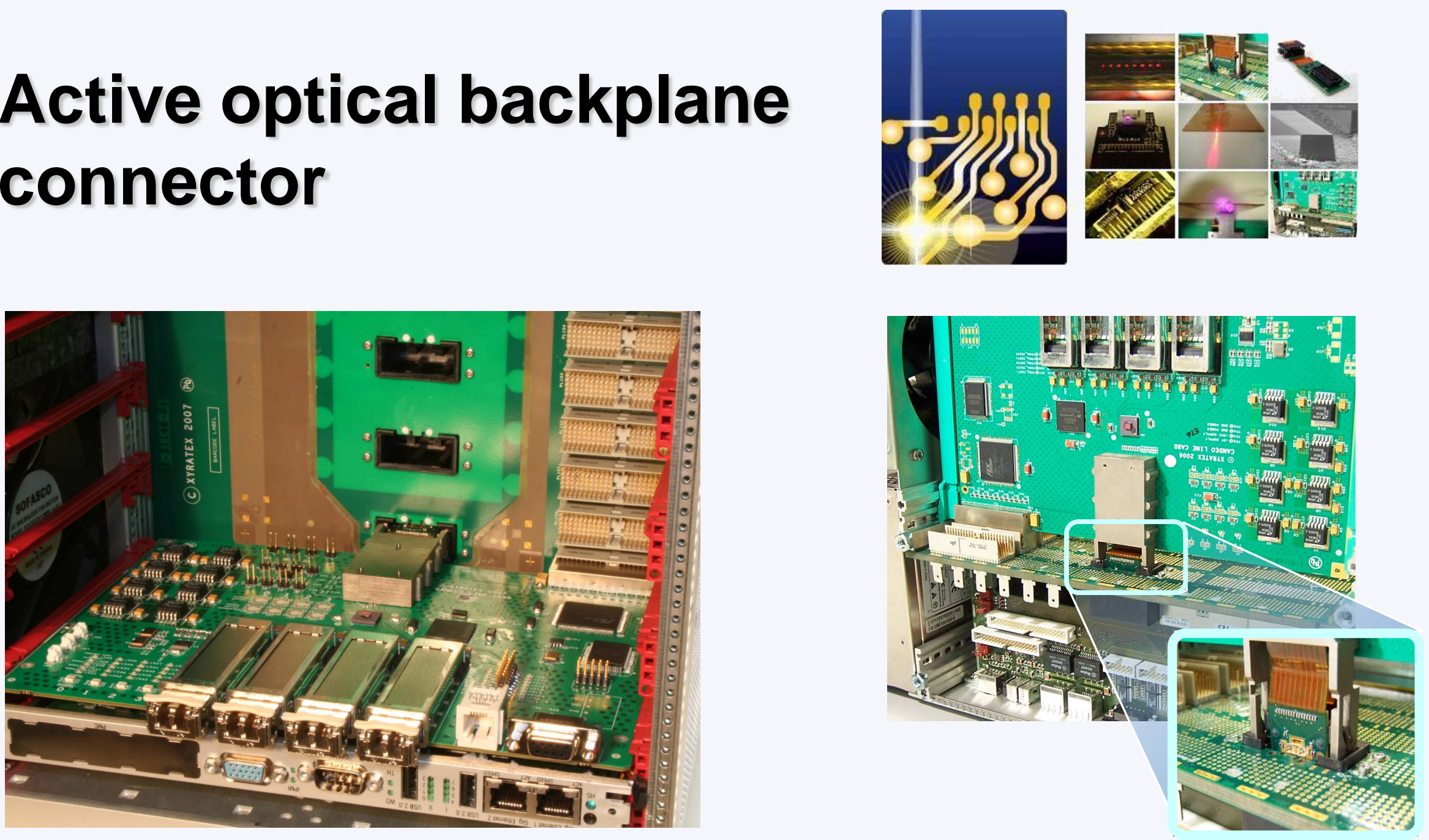


\section{Optical backplane connection architecture}
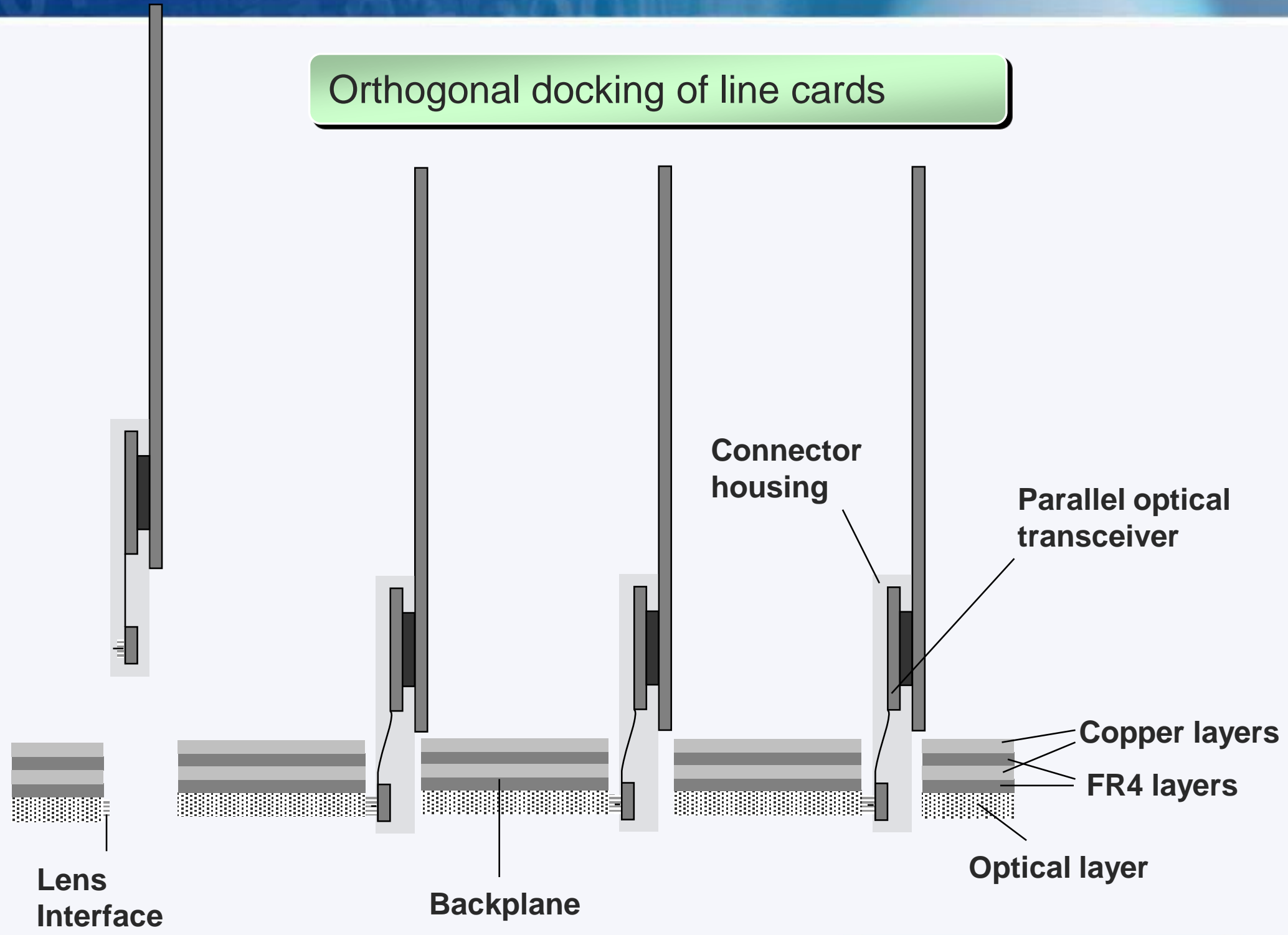


\section{Parallel optical transceiver}

- Mechanically flexible optical platform

MT compatible optical interface

a Geometric microlens array

- Quad VCSEL driver and TIA/LA

VCSEL / PIN arrays on pre-aligned frame
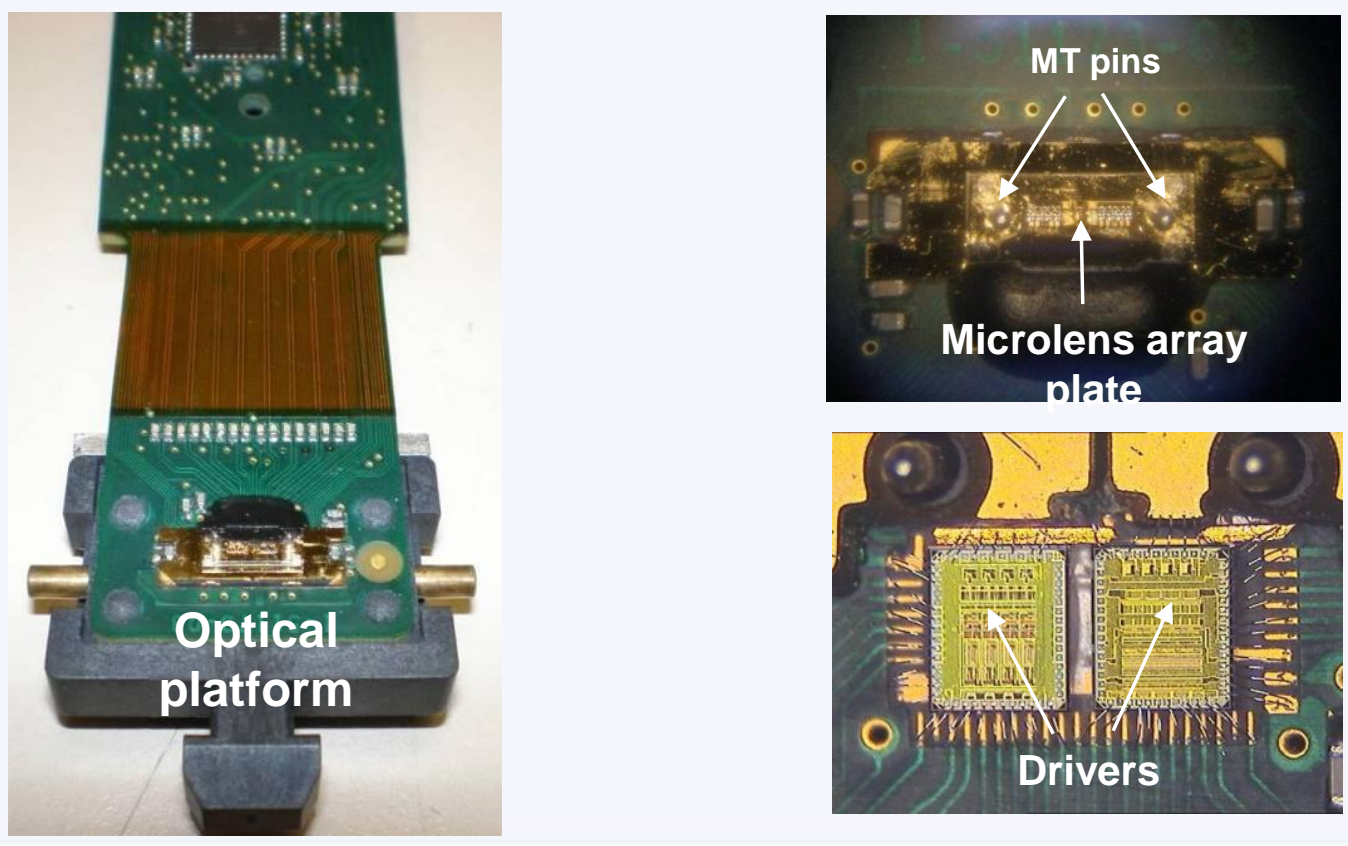

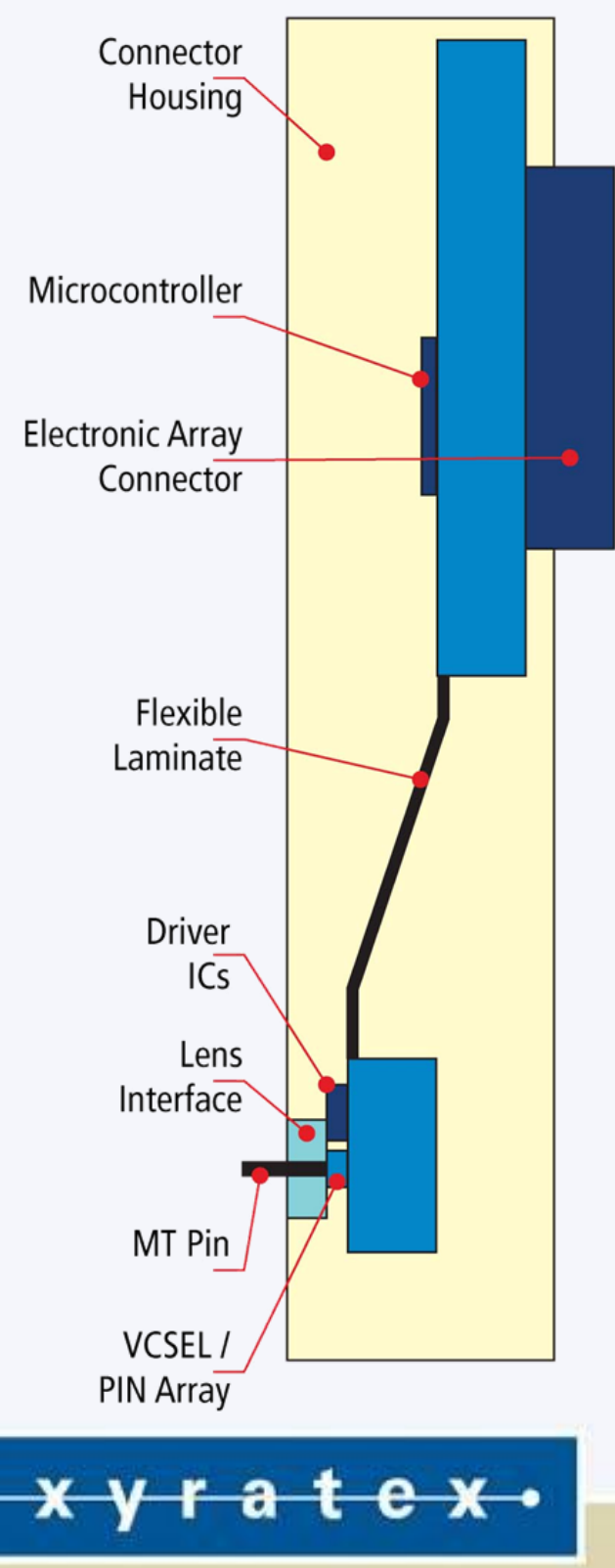




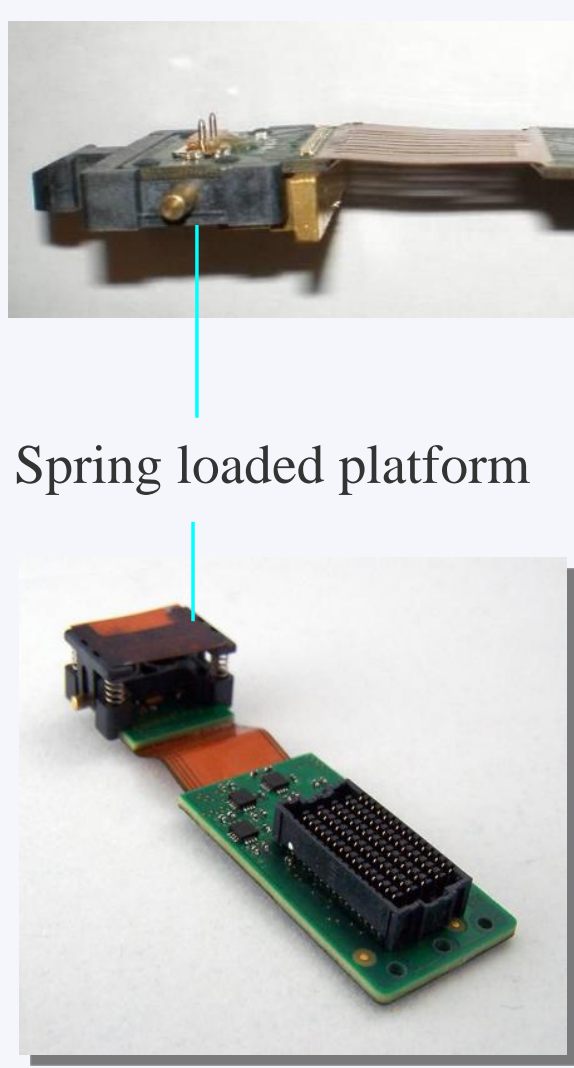

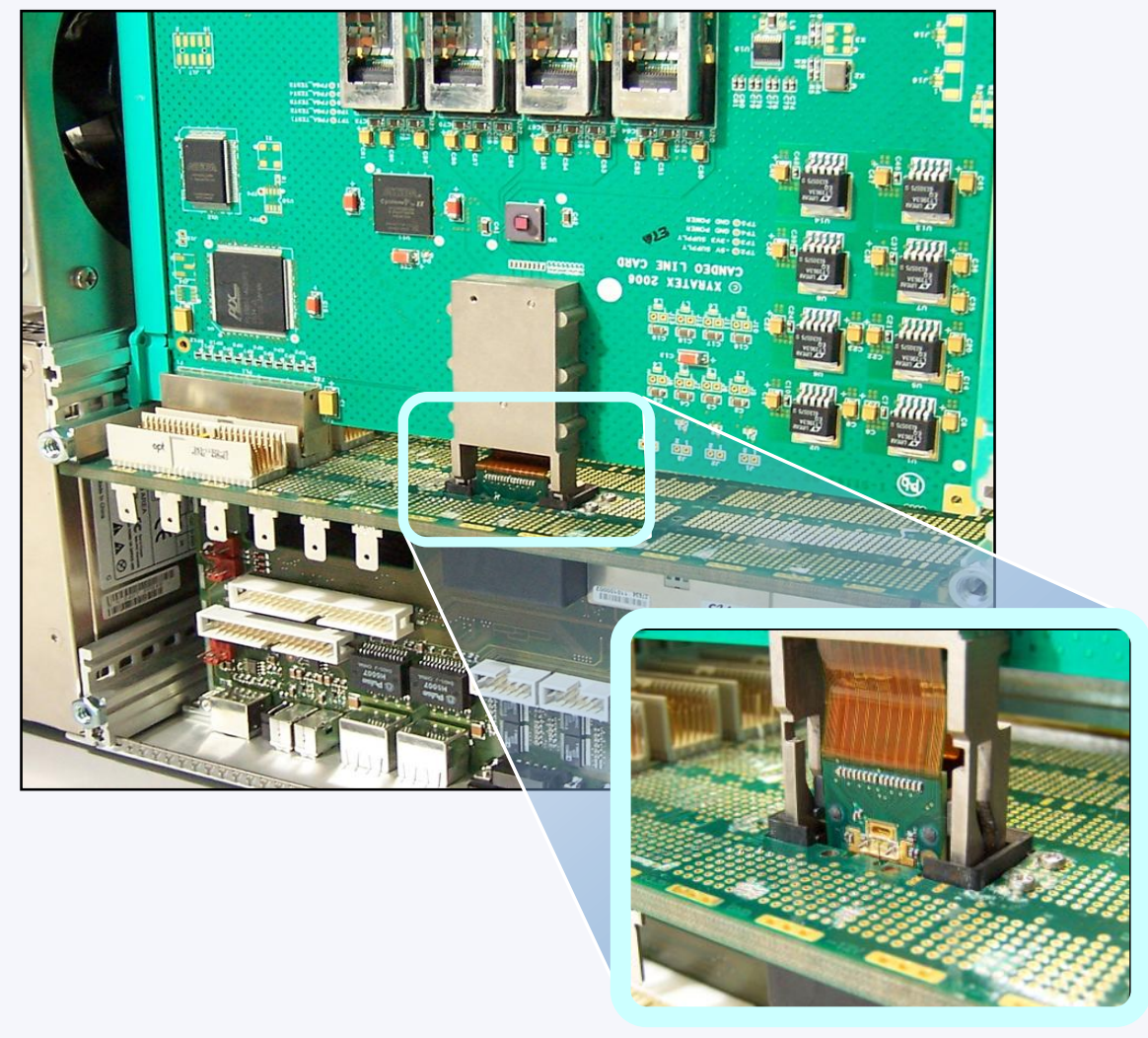

$x-y+a t-x$ 
Cam followers guided along cam track

- Allows for orthogonal movement of optical platform

- Ramped plug for reversible connection
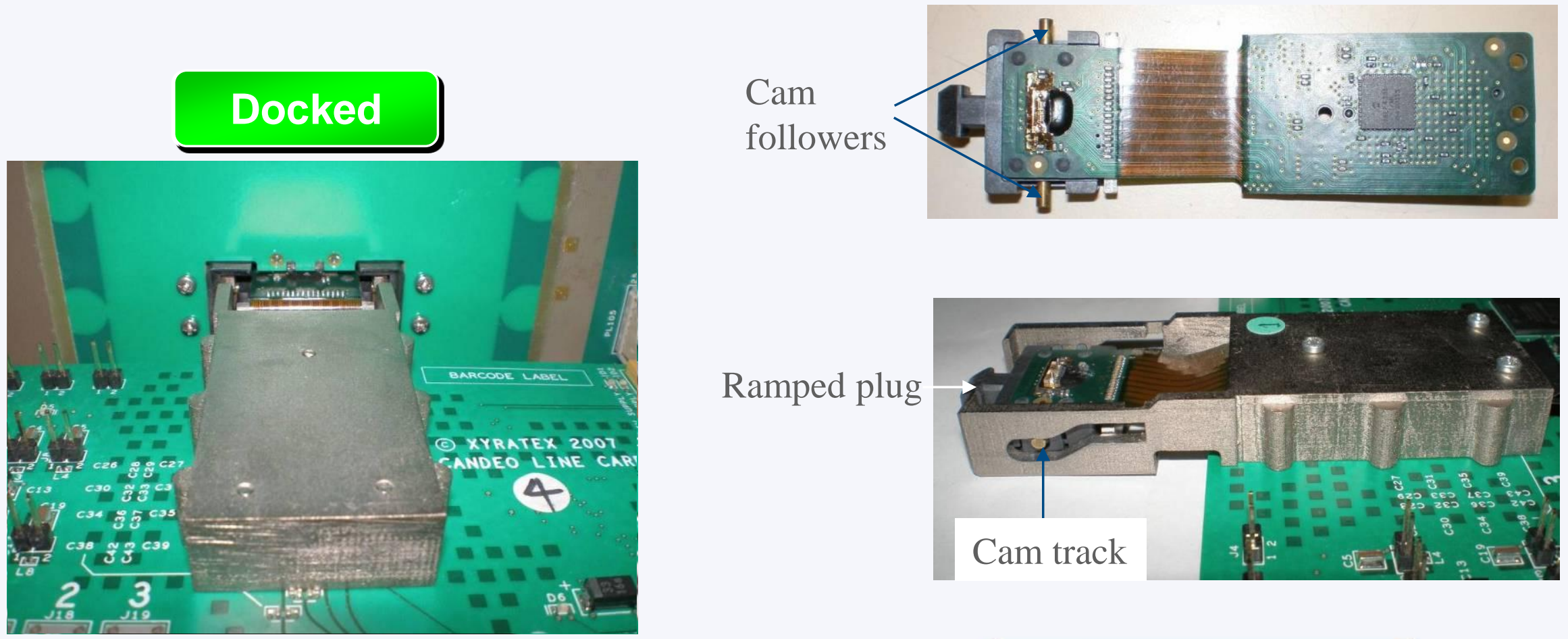


\section{Optical backplane connection architecture}

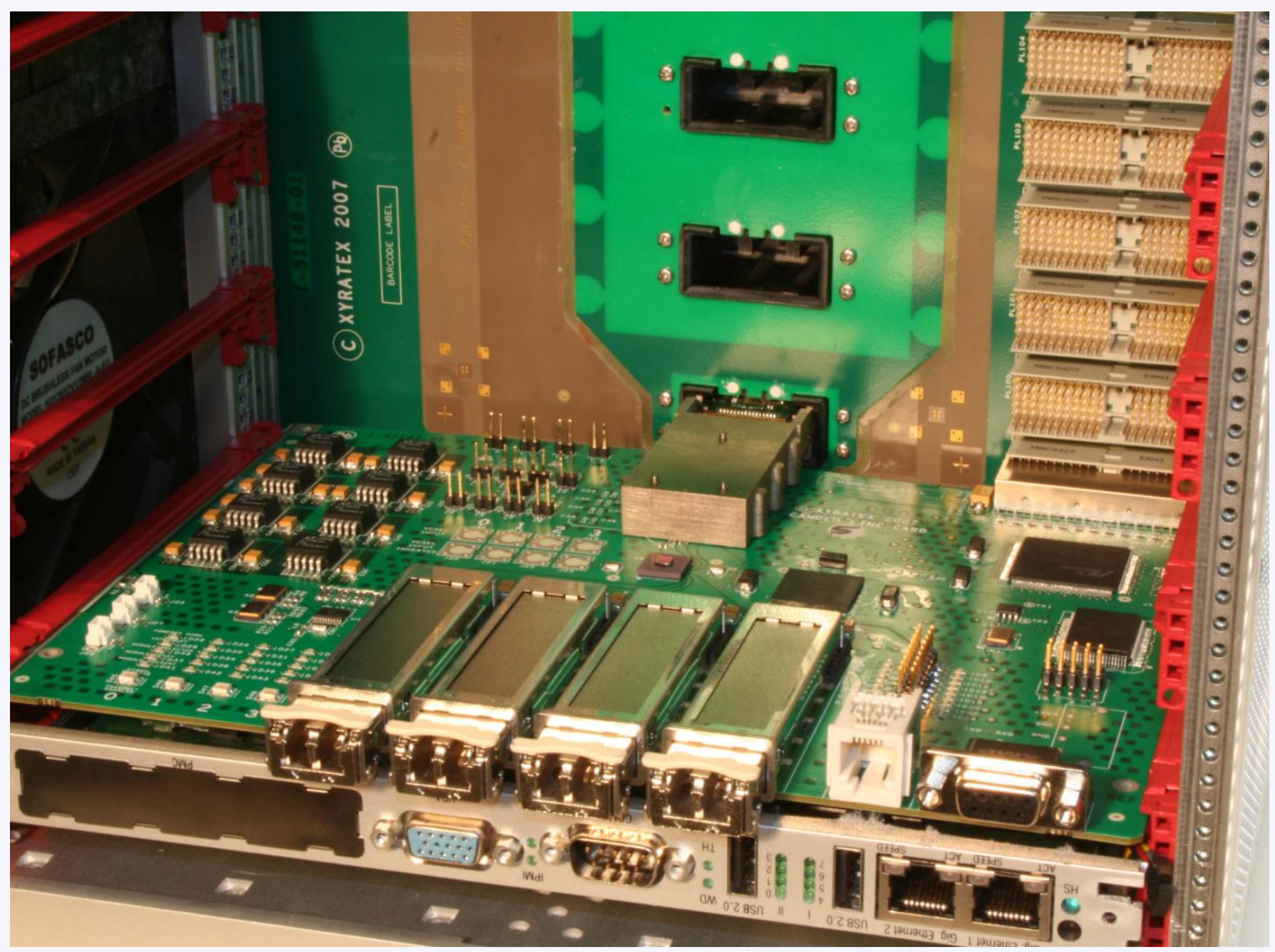




\section{Dual lens coupling interface}

\section{Free space coupling arrangement}

$\checkmark$ Optimised for loss minimisation

Maximum beam expansion

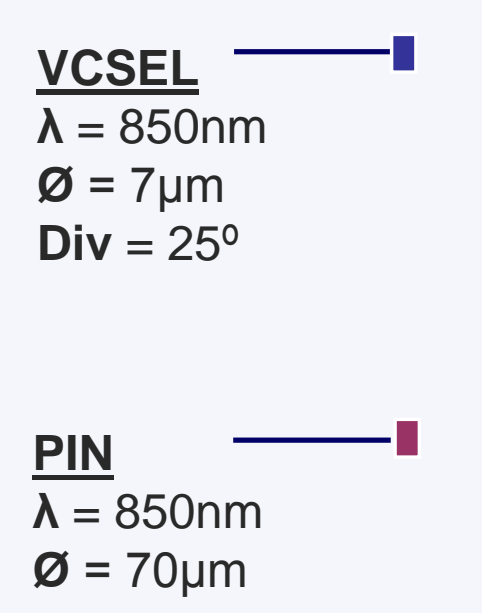

Predicted interface loss: $\mathbf{0 . 7 2} \mathrm{dB}$

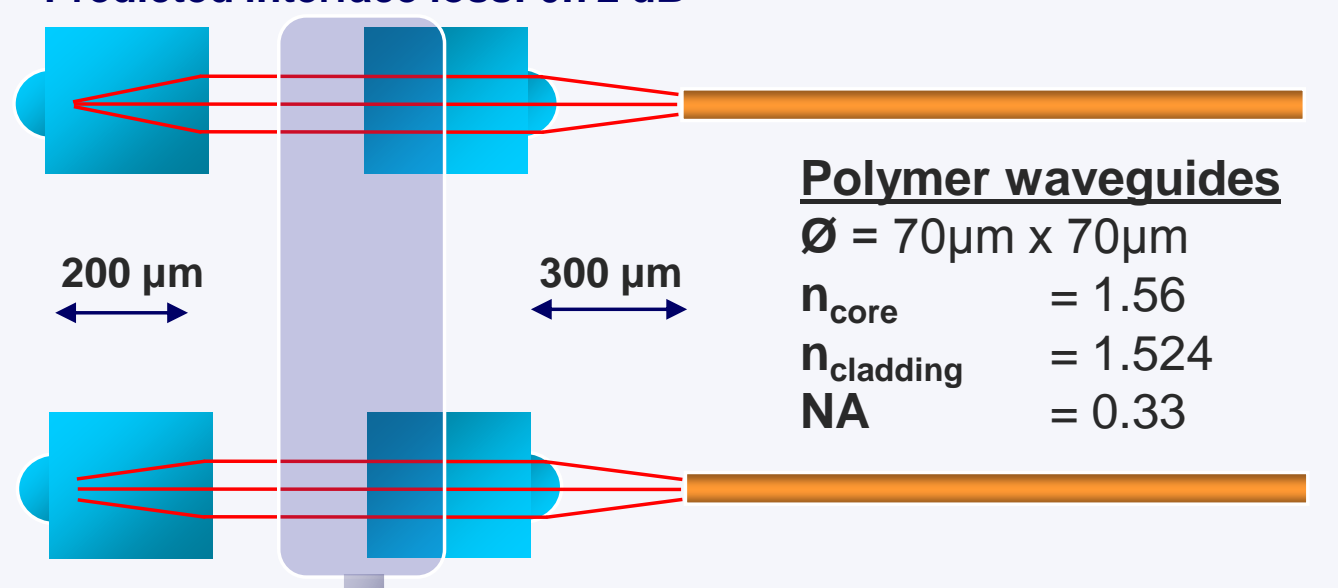

Predicted interface loss: $1.11 \mathrm{~dB}$

\section{Dual lens coupling solution}

$\checkmark$ Beam expansion at coupling interface

$\checkmark$ Reduces susceptibility to contamination 


\section{Demonstration assembly}




\section{Demonstration platform with peripheral test cards}

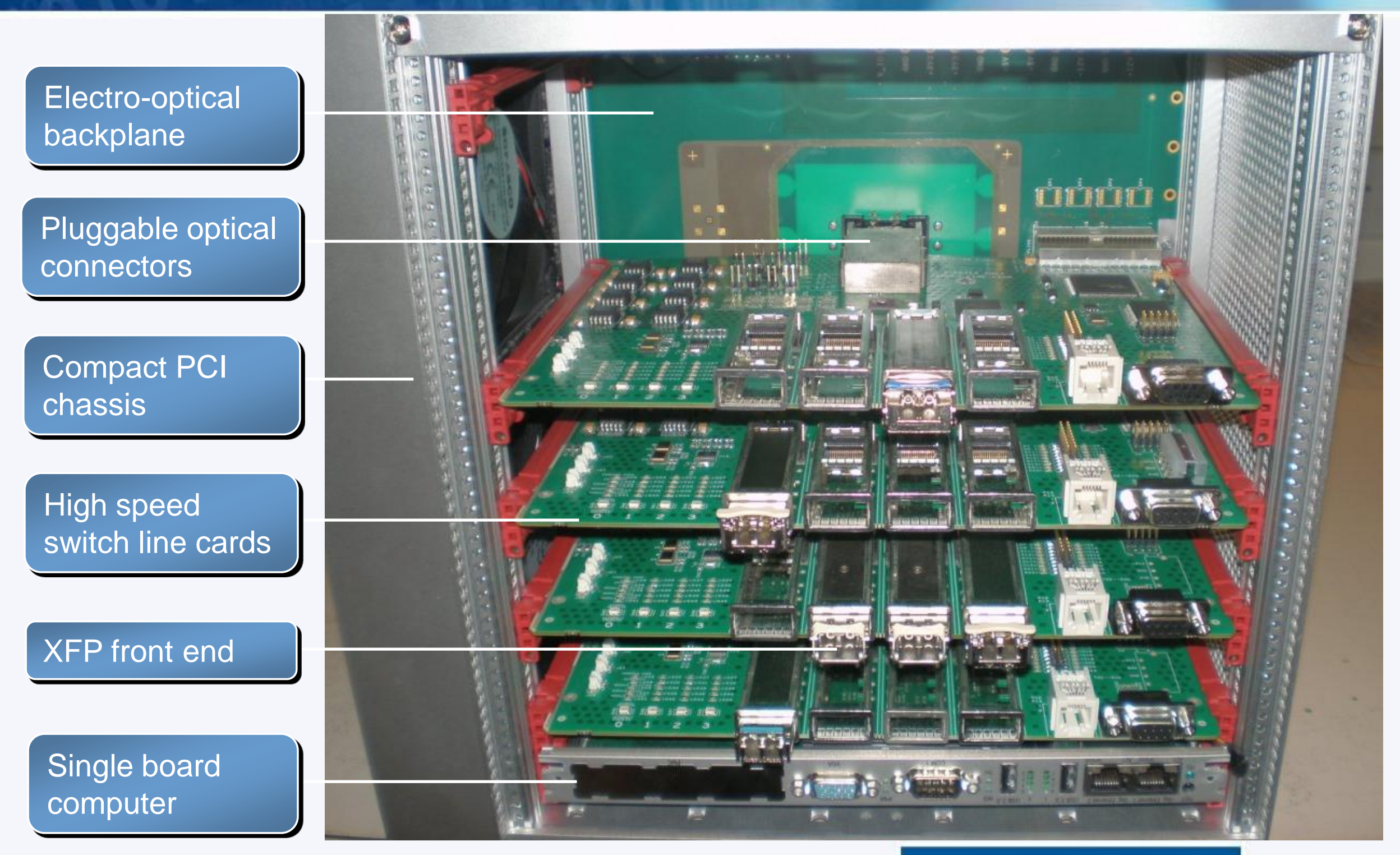




\section{Demonstration assembly}

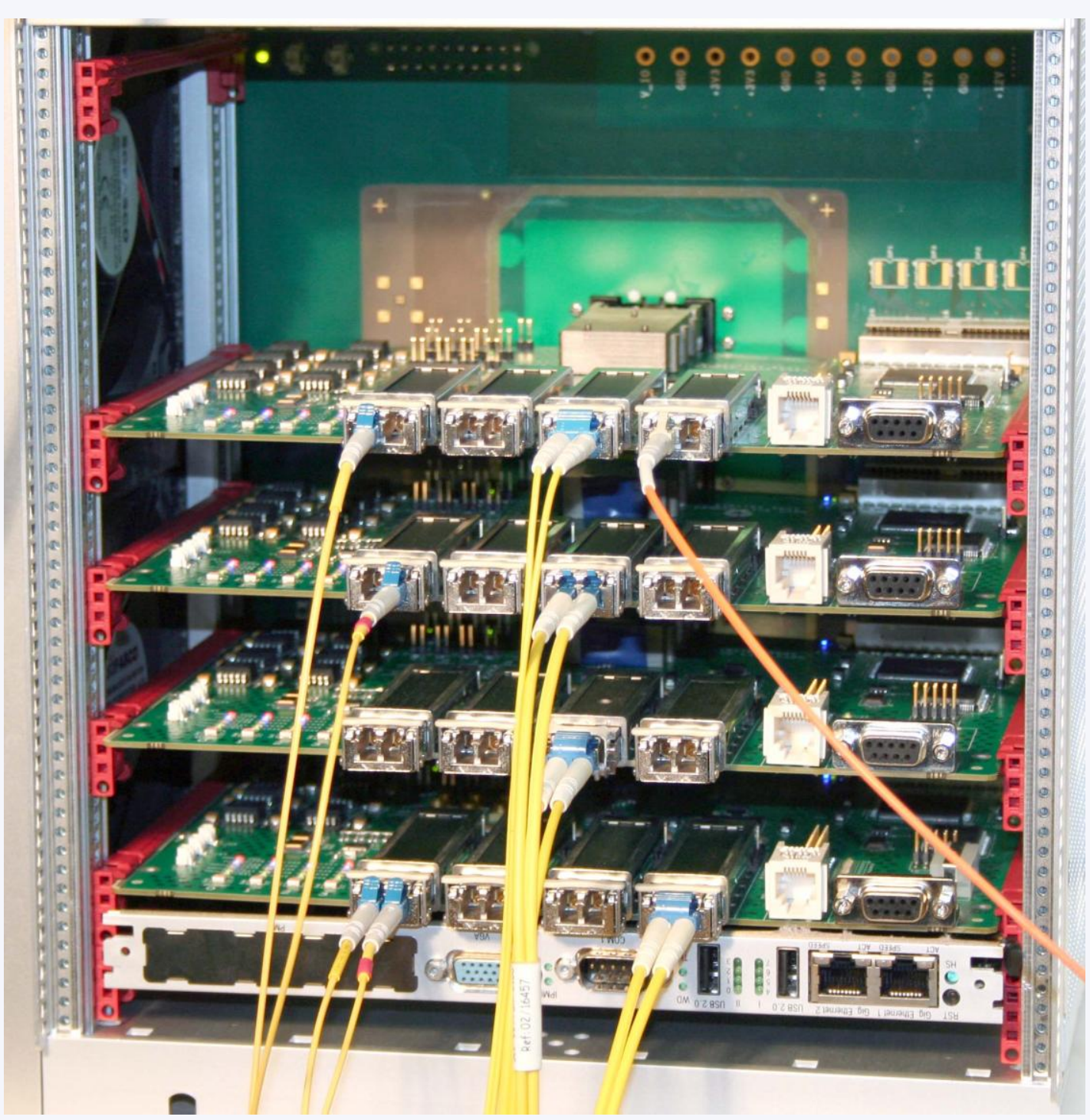

\section{Procedure}

10 GbE LAN test traffic @ $10.3 \mathrm{~Gb} / \mathrm{s}$ into demo front end ( $1^{\text {st }}$ line card)

․ Data passed across pluggable connectors and optical backplane

Data retrieved through front end of $2^{\text {nd }}$ line card

$$
x-y r a t e x
$$




\section{High speed data transmission measurements}

\section{Procedure}

口 $10 \mathrm{GbE}$ LAN test traffic @ $10.3 \mathrm{~Gb} / \mathrm{s}$ into demo front end ( $1^{\text {st }}$ line card)

D Data passed across pluggable connectors and optical backplane

Data retrieved through front end of $2^{\text {nd }}$ line card

\section{Results}

- Test data captured with typical peak to peak jitter 30ps (after front end CDR)

$\square$ Total optical waveguide interconnect loss ranges from $-6 \mathrm{~dB}$ to $-13 \mathrm{~dB}$

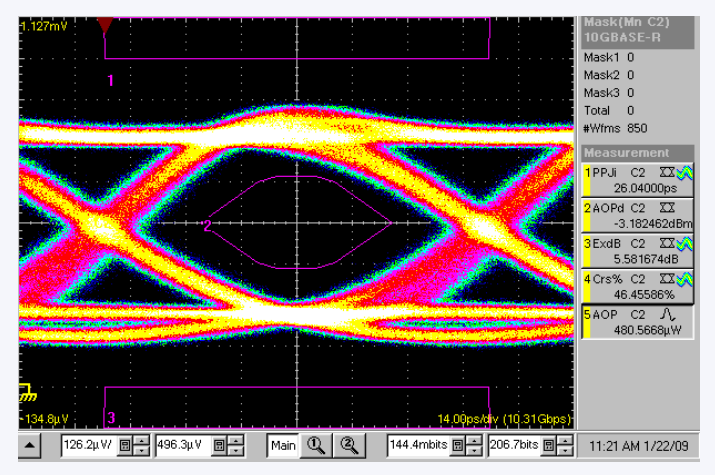

SPIE Photonics West 2010

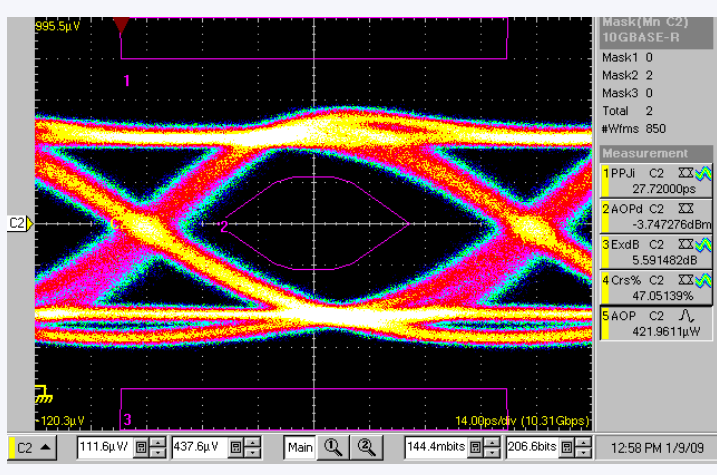

Opto: $7607-18$

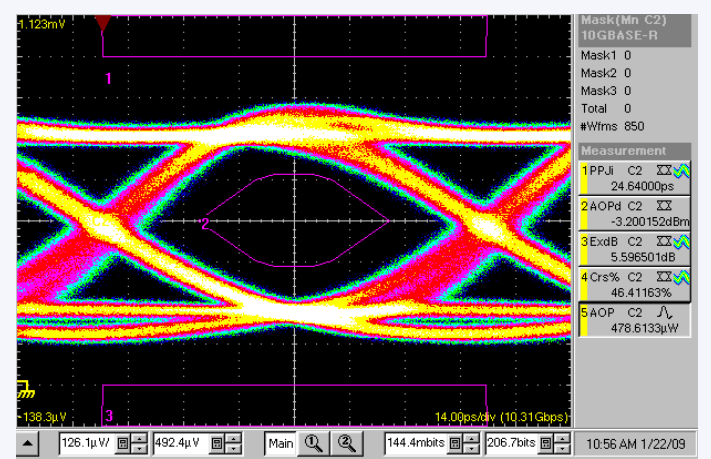

$x-y<a t-x$ 


\section{Acknowledgements}

Will write something here

\section{EPSRC}

DTI

Samtec 


\section{Thank you for your attention}

Richard Pitwon

Xyratex Technology Ltd

Langstone Road, Havant

Hampshire PO9 1SA

United Kingdom

E-mail: rpitwon@xyratex.com

\section{$\underline{\text { Authors }}$}

Richard Pitwon, Ken Hopkins

Kai Wang, David R. Selviah, Hadi Baghsiahi

Bert Offrein, Roger Dangel, Folkert Horst

Markus Halter, Max Gmür
Xyratex Technology Ltd

University College London

IBM Research GmbH

Varioprint AG 